\title{
Development of a Mechatronic System for Plant Recognition and Intra-row Weeding With Machine Vision
}

\author{
Nicole Berkmortel ${ }^{1}$, Matthew Curtis ${ }^{1}$, Colin Johnson ${ }^{1}$, Alicia Schmidt ${ }^{1}$, Elyse Hill ${ }^{1} \&$ S. Andrew Gadsden ${ }^{1}$ \\ ${ }^{1}$ College of Engineering and Physical Sciences, University of Guelph, Guelph, Ontario, Canada \\ Correspondence: S. Andrew Gadsden, College of Engineering and Physical Sciences, University of Guelph, \\ Guelph, Ontario, Canada. Tel: 1-519-824-4120. E-mail: gadsden@uoguelph.ca
}

Received: August 9, 2020

Accepted: January 28, $2021 \quad$ Online Published: February 15, 2021

doi:10.5539/jas.v13n3p1

URL: https://doi.org/10.5539/jas.v13n3p1

\begin{abstract}
In this paper, an electro-mechanical integrated weed management system was designed, constructed, and tested. This laboratory-scale solution integrated elements of machine vision, controls, and mechanical actuation to selectively remove weeds from within a crop row. The device was validated in a controlled environment using corn crops. Various crop conditions were considered to ensure the robustness of the design. Though some design aspects should be reworked for improved results, the device can effectively be used to facilitate small-scale research for automated weeding strategies.
\end{abstract}

Keywords: crop sciences, machine vision, mechatronics, weed management

\section{Introduction}

Herbicide resistance is currently a prominent issue in the agricultural community. Weeds such as Conyza Canadensis (e.g., horseweed) are resistant to herbicides due to frequent overuse. Although stronger chemical use can mitigate the problem, this approach is expensive and poses a significant risk to human health and environmental quality. As a result, companies are seeking to reduce herbicide dependence using technology that employs integrated weed management (IWM) strategies. IWM techniques use mechanical, computer, or a combination of both to address weeding. Traditional soil-cultivating tools are effective for removing weeds between crop rows (inter-row weeds), but risk damaging crops to remove weeds within crop rows (intra-row weeds). As intra-row weeds pose the most nutrient competition to crops, they are the target of current IWM strategies. The earliest IWM tools appeared in the 1960s and were purely mechanical. One example of a pure mechanical IWM is the Steketee finger weeder, which used fingered wheels to lightly till soil and uproot small intra-row weeds (Sutton Agricultural Enterprises, 2020). However, this machine and those similar cannot aggressively till the soil (as with inter-row weeds) due to potential damage to crops. Further, it cannot differentiate between crops and weeds, highlighting the downside to purely mechanical systems.

Recently, electro-mechanical systems have been developed to replace mechanical weeding machines. These tools use a combination of machine vision and control for automated intra-row weeding. Machine vision is used to differentiate between crops and weeds, while a mechanical tool is used to cultivate. Manufacturers such as Sutton Agricultural Enterprises and Garford Farm Machinery have developed and commercialized such systems for high-value cash crops (Bond \& Grundy, 2001). However, these solutions only exist on a commercial level, inhibiting testing of diverse solutions to IWM. Thus, this study sought to design and build a laboratory-scale cultivator for research and testing of automated, selective intra-row weeding technology. Driven by several design considerations, the system is based on a review of current machine vision techniques and a synthesis of various tooling strategies. Results indicate a functional design with promising future application.

There are a variety of mechanical tillage tools available for weeding. Straight blade designs include those of the Sarl Radis machine with linearly inserted and retracted blades or those of Steketee finger weeder with blades rotated in and out of rows (Upadhyaya \& Blackshaw, 2007). Green and Owen designed a novel rotating disk blade designed to provide clearance to crops as a machine passed through the rows (Green \& Owen, 2011). Experimental testing for small scale, automated weeding methods is typically conducted using soil bins and a tool trolley (Durant, Perumpral, \& Desai, 1980). A traditional soil bin set up includes a rectangular soil box representing the ground and a mobile tool carriage modelling tractor-drawn travel over soil. Unmanned systems have also been utilized in the last few decades in an effort to increase the efficiency of agriculture, in particular, 
by reducing labor needs (Bonadies \& Gadsden, 2019). These systems have been used for a variety of purposes including: soil sampling, irrigation management, precision spraying, mechanical weeding, and crop harvesting (Taylor, Charlton, \& Yúnez-Naude, 2012; Billingsley, Visala, \& Dunn, 2008; Vidoni, Bietresato, Gasparetto, \& Mazzetto, 2015). While research into unmanned systems in agriculture has been conducted over the last three decades, commercial products have been slowly adopted by farmers (Bonadies et al., 2017). However, due to recent epidemiological crises around the global, the use of autonomous systems for agriculture is expected to grow rapidly in the next few years as labor shortages are expected.

The goal of this study was to design a small-scale apparatus for testing research of automated IWM, with a focus on intra-row weeding. A review of the current state of automated intra-row weeding technology revealed design challenges that can be broken into four distinct categories: plant detection, position tracking, weeding tool design, and experimental testing. Modern weeding methods use machine vision to translate color and position from image pixels into information for additional computation. This has been explored in a variety of ways. For example, the authors in (Åstrand \& Baerveldt, 2002) designed a system to identify and classify sugar beets and weeds by identifying the color and shape features of both. Alternatively, Green and Owen in (Green \& Owen, 2011) employed blob detection on cabbage rows. By finding areas where pixel properties were consistent this method effectively separated crops from the image background. After plant detection, the position of the plant relative to the weeding tool must be determined and tracked. Studies such as (Åstrand \& Baerveldt, 2002) and (Bontsema, van Asselt, Lempens, \& van Straten, 1998) have shown implementing encoders mounted on wheels to track tool position relative to the detected plan have been successful. From the review of current methods, five design considerations were outlined for the construction of the testing apparatus. The machine should be able to: 1) detect the location of an approaching crop; 2) monitor the location of a crop relative to the weeding tool; 3 ) control the weeding tool; 4) actuate the weeding tool; and, 5) move to simulate field operation. This paper is organized as follows. The design process and experimental setup is described in Section 2. The experiment setup and results are stated and discussed in Section 4. The paper is then concluded and future recommendations are provided.

\section{Materials and Methods}

In this section, environmental conditions, system design, and the experimental design for the small-scale apparatus for testing IWM are considered.

\subsection{Environmental Conditions}

This study considered corn, a common cash crop, as the plant of interest. As such, the environmental conditions surrounding corn growth and development governed the design process and testing. Design of the IWM apparatus was based on a mixture of realistic and ideal environmental factors, which was deemed acceptable as the intended use is for continuous research. Five environmental factors were considered: growth parameters, crop spacing, soil conditions, temperature conditions, and light intensity. Growth parameters refer to the rate of emergence for the crop. Corn seeds are normally planted at a depth of $2-4 \mathrm{~cm}$ below the soil surface and follow the early growth stages defined in Table 1 . Weed removal is critical at the V3/V4 stage of development in order to prevent nutrient competition of the more developed root system, making this stage the focus of the experiment (Ransom \& Endres, 2014).

Table 1. Corn growth parameters considered in the study

\begin{tabular}{ll}
\hline Growth Stage & Timeline \\
\hline VE & $3-4$ weeks post germination \\
V1/V2 & 1 week post above ground emergence \\
V3/V4 & 2 weeks post above ground emergence \\
\hline
\end{tabular}

Crop spacing refers to the amount of intra-row crop spacing. The selected spacing for this experiment was $61 \mathrm{~cm}$, which is larger than the average corn spacing of $18 \mathrm{~cm}$. Soil conditions refer to the composition and density of the soil, which range widely across Ontario. This experiment employed PGX Pro-Mix for the soil medium because it had ideal nutrient retention capabilities. The soil was loosely packed into the soil bins as opposed to compacted like a real farm. Temperature conditions refer to the impact of seasonal changes on the growing temperature of the crops. The normal growing season for corn in Ontario is May to October, during which the average amount of daylight is 13-15 hours (Ransom \& Endres, 2014). Artificial temperature conditions were selected as 16 hours of daylight with daytime temperature of $25^{\circ} \mathrm{C}$ and nighttime temperature of $20^{\circ} \mathrm{C}$, which 
were more ideal than realistic. Light intensity refers to the brightness level of the lights in the testing facility. Tests were conducted at the Crop Science Building of the University of Guelph. This facility was equipped with a mix of LED and incandescent light bulbs that created a well-illuminated environment. Again, this was deemed an acceptable deviation from realistic conditions in which light intensity would fluctuate severely.

\subsection{System Design}

The IWM prototype was intended to present an electro-mechanical solution using traditional tillage combined with mechatronic techniques. The mechanical systems were designed first, and centered around the design of the weeding tool and a mechanical structure for the device. The weeding tool required a means of actuation and a design for its end effector. Rotary actuation in similar style to (Green \& Owen, 2011) was employed because it permitted a tool to directly connect to a motor output shaft. A NEMA 34 stepper motor and driver were selected for use with this method of actuation because of their low cost and ease of control. The authors in (Green \& Owen, 2011) also inspired the use of a disc-shaped tool for the end effector. This disc has a cut out "safe region", a circular area of clearance around the crop stem that is included to compensate for potential error between the camera sensor and the actual location of a plant. In this study, the design was modified to include two serrated discs, one disc on either side of a crop, for better ground coverage. The tillage depth of the end effector was constrained by the corn seed planting depth of $2-4 \mathrm{~cm}$. Based on this, a tillage depth of $2 \mathrm{~cm}$ or less was selected. This would allow the device to remove weeds with shallow root systems but not compromise the crops' root systems. The diameter of the blades was determined through trial and error, and was selected as $15 \mathrm{~cm}$ with a 7.5 cm safe region.

Traditional soil bin tests use mobile carriages. Thus, a rolling trolley design was implemented for the mechanical structure of the prototype. The design was physically constrained by the dimensions of the soil bin and the space constraints of the Crop Science facility at the University of Guelph. Vibration reduction and tool modulation were also considered in the design. Minimizing vibration was critical to ensure image quality of the machine vision sensor. To combat this, a four wheel, "straddling" trolley with rubber-coated wheels was employed. Tool modulation was considered to allow for an adjustable tool height in case of variations in soil level. Height adjustment was implemented using a pinned sleeve design, where the lowest pin places the tool at a maximum of $2 \mathrm{~cm}$ below the soil. The highest pin places the tool well above the expected soil height for transportation purposes.

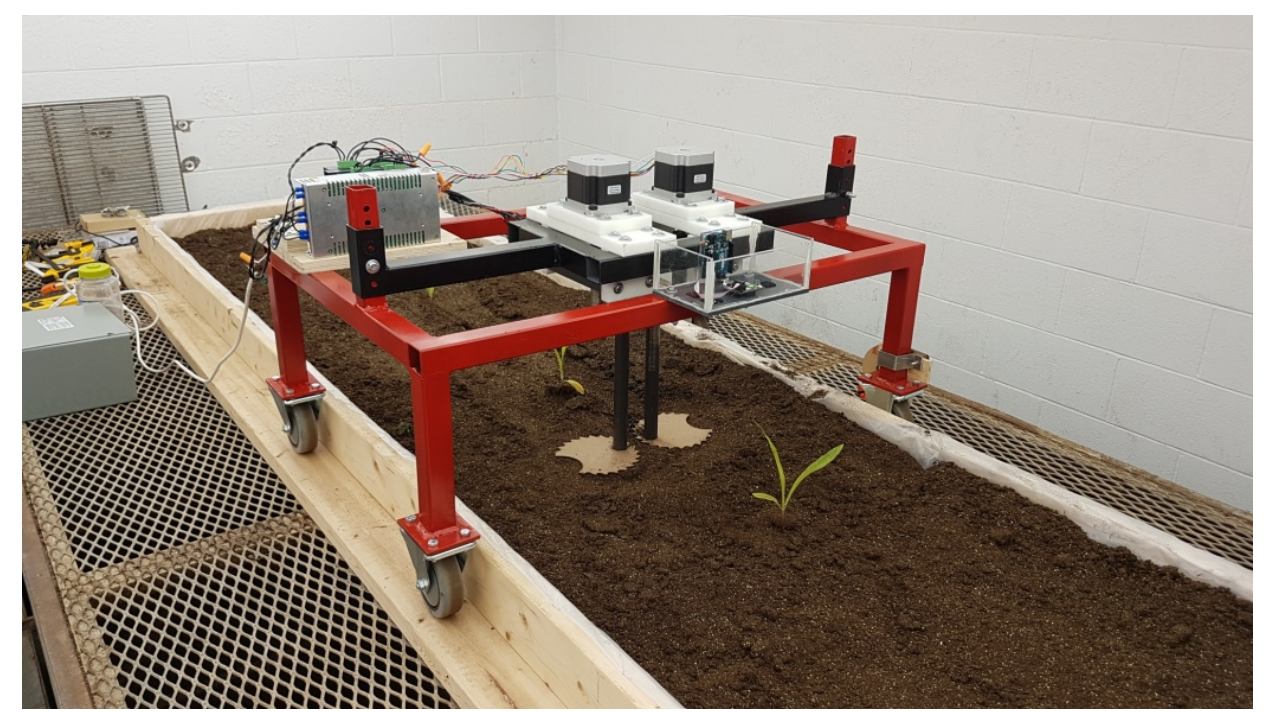

Figure 1. Final IWM device assembly in use at the indoor testing facility with corn plants

The mechatronic system, as shown in Figure 1, centered on the selection, programming, and integration of a camera system and a position tracking system. A CMUcam5 Pixy was selected as the machine vision sensor. The Pixy is programmable with XCTU and Pixymon firmware. Vision-based weeding can take two main approaches: crop and weed detection, distinctly identifying both plants, or pure crop detection, distinguishing only crop and not-crop areas. Pure crop detection was employed on the Pixy, programmed to recognize a corn crop based on its size and location in an image. The above ground stages of growth were programmed as "target descriptions", or 
the ideal value, of corn crop size. Thus, the size criteria determined if a plant detected in the image was of adequate size to be considered corn based on these values. If not of target size, a plant was regarded instead as a weed. The location of a plant in an image also factored into crop detection. Modern precision planting allows planters to have a rough idea on the location of the next expected crop, allowing an expected region of interest to be programmed into the software. This permits only plants in this region to be recognized as crops and all plants outside the zone to be considered as weeds. An example of this is displayed in Figure 2 which labels a Corn Detection Region.

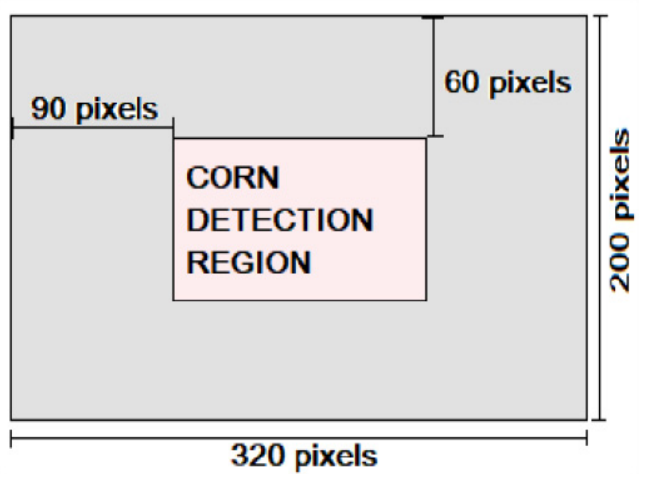

Figure 2. Screen partitioning of corn detection region

Position tracking was also used to actuate the weeding tool. A rotary encoder was selected to track the progress of the trolley as it approached a crop, thereby providing position information. Mounted to the wheels of the trolley, the encoder measured wheel rotations in relation to crop detection, allowing the system to determine trolley distance from crops. This information was passed to a control algorithm which maneuvered the end effector from an unactuated to actuated position based on the Pixy image capture, as shown in Figure 3. Once a crop was recognized, the algorithm counted encoder ticks until it reached a set value representing full actuation of the end effector. As the trolley passed over the crop, it would return to its unactuated position. To improve end effector actuation, the motor resolution, pulses per step, and delay were changed as necessary. Motor resolution represents the number of steps the motors take to rotate 360 degrees, pulses per step represents the amount of encoder units between each step of the motors, and the delay represents the amount of encoder units to wait between plant detection and the blades reaching the plant. Control was implemented using two Arduino Uno microcontrollers, one for obtaining the image and encoder inputs and the other to send pulse width modulation signals to the motor drivers. Figure 4 represents the flow diagram of the implemented control algorithm. 


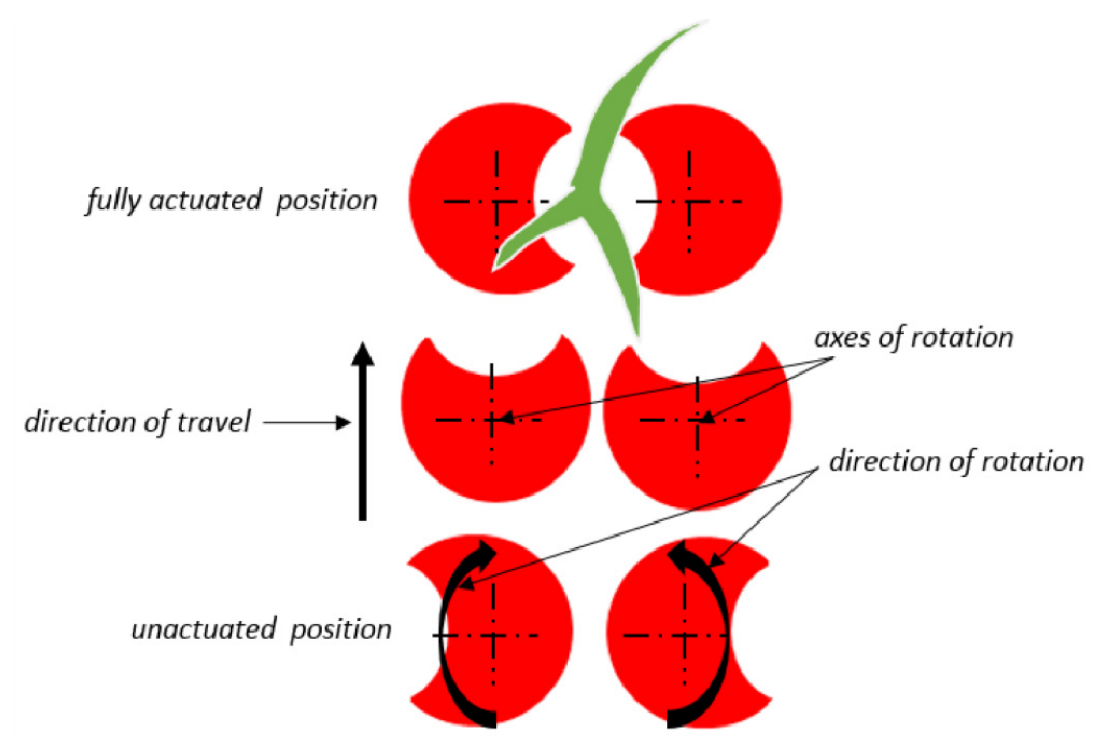

Figure 3. Actuation of designed end effector

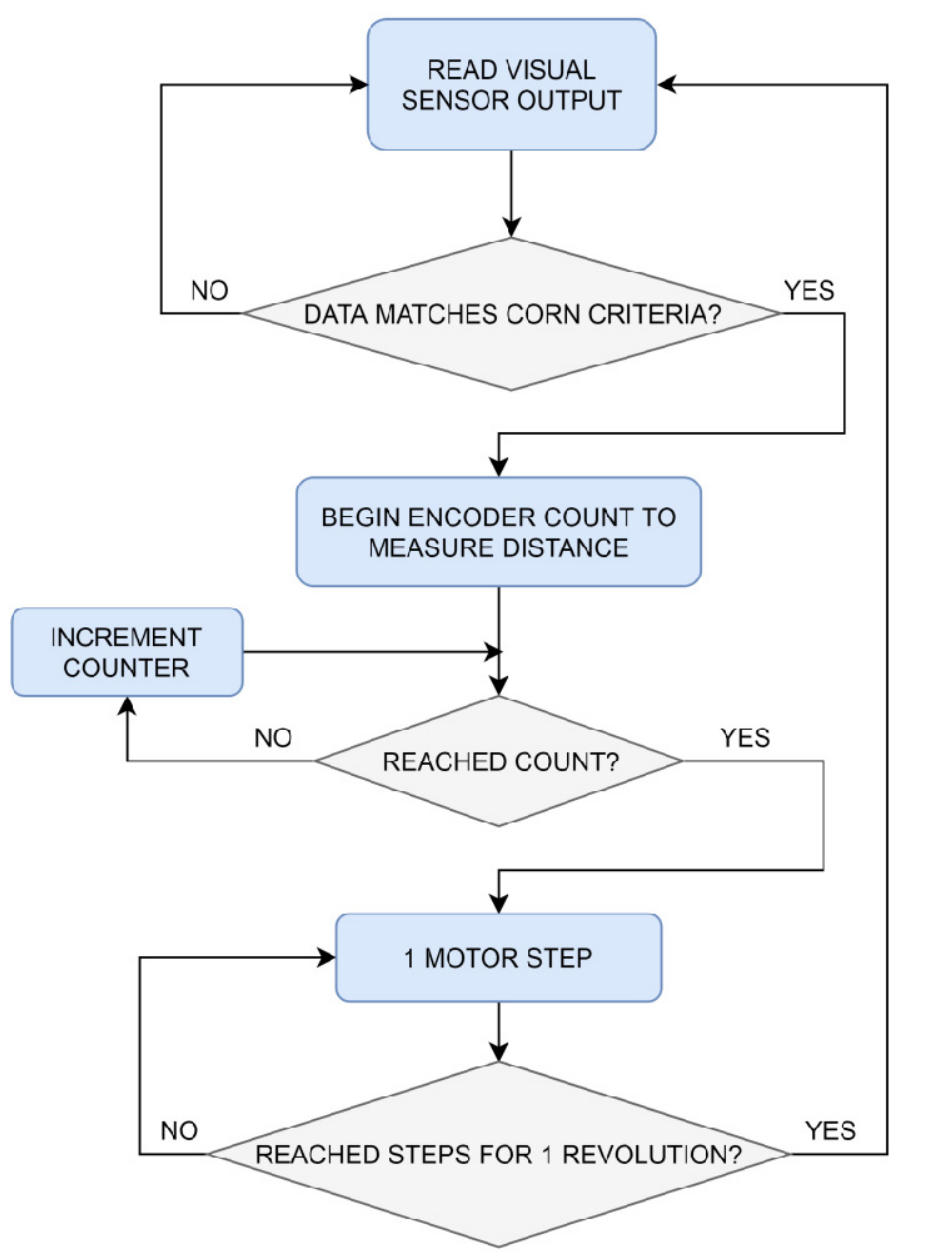

Figure 4. Flow diagram represented the implemented control algorithm 


\subsection{Experimental Design}

To verify the functionality of the IWM device, tests were conducted with soil bins that measured $1 \mathrm{~m}$ in width at the Crop Science facility. The goal of each test would be to determine if a crop was safe: did it survive the automated weeding process. Based on the results, aspects of the control algorithm would be changed until performance was satisfactory. No weeding was conducted in the experiments, so the effectiveness of the design on weed removal could not be measured. Testing occurred by rolling the IWM assembly over the soil bed and measuring the reaction to the stimuli. When the trolley was above a corn crop, the position of the blades with respect to the plant stem was measured as shown in Figure 5a. If the distance from the plant stem to the back tip of the blade crescent was within the concave portion of the blade (the safe region), the plant was marked as safe and given the binary value of "Yes". If the plant stem was outside this distance, it was marked as unsafe and given the binary value of "No". Based on a minimum of three trials of binary data, the motor resolution, pulses per step, and delay parameters of the control algorithm were changed to improve performance. Thus, the data collected are hit-miss values for plant survival and controller parameters for satisfactory trials.
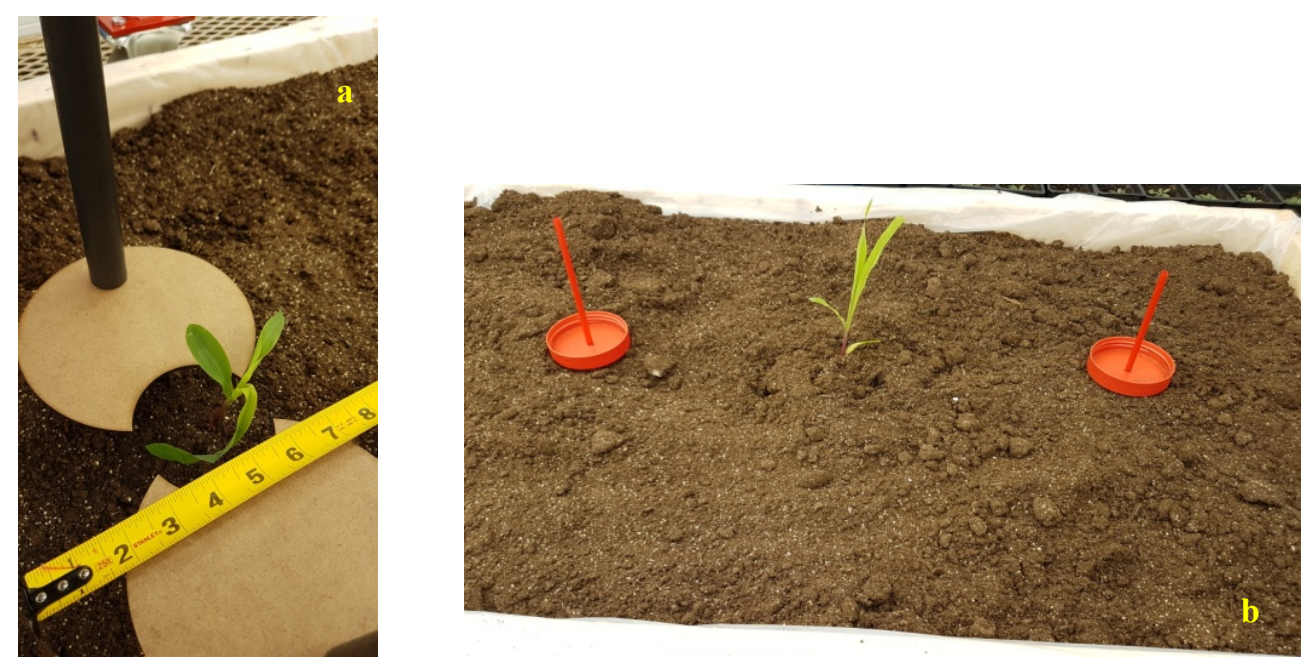

Figure 5. Testing environment for manual measurements and calibration of system

Note. a: Crop survival measurement. b: Red cup lids used for calibration.

The IWM device was tested in four scenarios: Benchmark, Best Case, Spacing, and Bounds. The Benchmark test was used to ensure proper functionality and calibrate initial control parameters. Red cup lids, displayed in Figure $5 \mathrm{~b}$, were used in place of corn crops for this test because they had uniform shape and size, making them ideal for calibration purposes. The Best Case scenario represented an ideal crop harvesting situation, with corn crops at $\mathrm{V} 3 / \mathrm{V} 4$ growth and intra-row spacing of $61 \mathrm{~cm}$. The Spacing and Bounds cases each represented deviations from the Best Case. In the Spacing tests, intra-row spacing was varied to imitate cases where there is increased or decreased distance between crops. The increased spacing, deemed Spacing-Increased, represents when a crop does not sprout or dies, resulting in larger spaces than expected in crop rows. Alternatively, Spacing-Decreased scenarios imitate when crops are planted closer together to increase crop yield. Intra-row spacing for Spacing-Increased remained $61 \mathrm{~cm}$ but plants were removed from the row to simulate increased spacing. Spacing for Spacing-Decreased scenario was $30.5 \mathrm{~cm}$. The Bounds test was used to determine the maximum and minimum plant size detectable by the machine vision algorithm. This is important for determining the impact of plant size on weeding actuation, as there are cases where harvested crops may be at varying stages of development. To conduct this test, plants at different growth stages were placed in the soil bed. Table 2 presents the intra-row distances and growth stages of each scenario. 
Table 2. Corn growth parameters considered in the study

\begin{tabular}{lll}
\hline Scenario & Intra-Row Distance (cm) & Crop Growth Stage \\
\hline Benchmark & 61 & $\mathrm{~N} / \mathrm{A}$ \\
Best Case & 61 & $\mathrm{~V} 3 / \mathrm{V} 4$ \\
Spacing-Increased & 61 & $\mathrm{~V} 3 / \mathrm{V} 4$ \\
Spacing-Decrease & 30.5 & $\mathrm{~V} 3 / \mathrm{V} 4$ \\
Bounds & 61 & $\mathrm{~V} 1 / \mathrm{V} 2, \mathrm{~V} 3 / \mathrm{V} 4$, beyond \\
\hline
\end{tabular}

\section{Results and Discussion}

The results of each scenario are presented in Tables 3-6 and the final apparatus control parameters for each scenario are found in Table 7. There is no hit-miss data recorded for the Spacing-Increased scenario, to be explained shortly. As seen, the Best Case controller specifications required minimal modification from the Benchmark calibration. Across the five trials, all specimens survived the weeding maneuver, highlighting the apparatus can function in an "ideal" scenario. The results of the Spacing and Bounds scenarios indicate the device's potential robustness. No data was recorded for the Spacing-Increased scenario because the significant increase in plant distance resulted in the motors not activating between plants, which is expected. However, the Spacing-Decreased case emphasized the limitations of the actuators, shown by the decrease in success rate. In trials with "missed" plants, it was observed that the motors had not finished their actuation cycle from the previous plant prior to reaching the subsequent crop. As such, controller parameters need to be further adjusted to ensure the blades return to an unactuated position so as not to kill the next encountered crop. The Bounds scenario revealed that the minimum size plant recognizable by the machine vision algorithm is subjective. As the Pixy can detect small resolution objects, it is up to the user to determine the lowest number of pixels for which a crop can be recognized. Here that size was set to $7.5 \mathrm{~cm}$ tall after multiple Bounds trials. This scenario also revealed the maximum size plant recognizable to be $28 \mathrm{~cm}$. Past this height, the machine vision algorithm correctly disregarded larger plants as viable crops because they failed to fit into the detection region, indicated by the misses on Plant 5 across all trials. The detection region can also be subjective because its design was based on corn growth stages, indicating that different upper bounds can be determined based on the desired acceptable growth stage. However, significantly increasing the crop detection region could undermine its purpose by introducing more crops into the field of vision or increasing processing time of the algorithm.

Table 3. Red cup calibration

\begin{tabular}{lllll}
\hline Trial & Plant 1 & Plant 2 & Plant 3 & Plant 4 \\
\hline Trial 1 & Yes & Yes & Yes & Yes \\
Trial 2 & Yes & Yes & Yes & Yes \\
Trial 3 & Yes & Yes & Yes & Yes \\
Trial 4 & Yes & Yes & Yes & Yes \\
Trial 5 & Yes & Yes & Yes & Yes \\
\hline
\end{tabular}

Table 4. Results of best case scenario

\begin{tabular}{lllll}
\hline Trial & Plant 1 & Plant 2 & Plant 3 & Plant 4 \\
\hline Trial 1 & Yes & Yes & Yes & Yes \\
Trial 2 & Yes & Yes & Yes & Yes \\
Trial 3 & Yes & Yes & Yes & Yes \\
Trial 4 & Yes & Yes & Yes & Yes \\
Trial 5 & Yes & Yes & Yes & Yes \\
\hline
\end{tabular}


Table 5. Results of pacing-decreased scenario

\begin{tabular}{llll}
\hline Trial & Plant 1 & Plant 2 & Plant 3 \\
\hline Trial 1 & Yes & Yes & No \\
Trial 2 & No & Yes & Yes \\
Trial 3 & Yes & Yes & Yes \\
Trial 4 & Yes & Yes & Yes \\
Trial 5 & Yes & No & Yes \\
\hline
\end{tabular}

Table 6. Results of bounds scenario

\begin{tabular}{llllll}
\hline Trial & Plant $\mathbf{1}$ & Plant 2 & Plant 3 & Plant 4 & Plant 5 \\
\hline Trial 1 & Yes & Yes & Yes & Yes & No \\
Trial 2 & Yes & Yes & Yes & Yes & No \\
Trial 3 & Yes & Yes & Yes & Yes & No \\
\hline
\end{tabular}

Table 7. Control parameters used in the scenarios

\begin{tabular}{llll}
\hline Scenario & Motor Resolution & Pulses Per Step & Delay \\
\hline Benchmark & 18 & 44 & 120 \\
Best Case & 18 & 44 & 140 \\
Spacing-Increased & N/A & N/A & N/A \\
Spacing-Decreased & 18 & 42 & 300 \\
Bounds & N/A & N/A & N/A \\
\hline
\end{tabular}

Results indicate the IWM apparatus functions sufficiently recognizing and not tilling crops. However, the testing conditions undeniably assisted in its effectiveness. Though testing conditions were intended to mimic reality, in practice they differed significantly regarding crop spacing, soil density, and light exposure. Crop spacing was much greater than average crop conditions at $61 \mathrm{~cm}$ row spacing as compared to the $18 \mathrm{~cm}$ average. This would significantly affect the image sensor's detection capabilities and the activation of the motors. The soil density was loose instead of compacted in order to allow the blades to move easier through the soil. Typical soil mediums are denser, which causes not only additional resistance to the blades but also soil to clump on tools, potentially damaging root systems of crops. Thus, the balance of soil resistance and root safety would need to be considered in blade design and actuator selection. Finally, the light exposure to the soil beds was constant in intensity and direction. Light was provided directly overhead test bins, eliminating the presence of shadows from testing. Varying light exposures would need to be considered to evaluate their impact on the image detection software, which would undoubtedly deteriorate in the presence of shadows.

\section{Conclusions}

A laboratory-scale, integrated weed management device was designed, built, and tested on intra-row weeding scenarios for a corn plant. Using an electro-mechanical approach, a control algorithm was designed and integrated with vision sensors to mechanically actuate stepper motors via encoder readings. Experiments verified the effectiveness of the prototype, which successfully identified crops and avoided uprooting them under a variety of conditions. Future work includes testing under increasingly rigorous scenarios that mimic real world harvesting and environmental conditions.

\section{References}

Åstrand, B., \& Baerveldt, A. (2002). An Agricultural Mobile Robot with Vision-Based Perception for Mechanical Weed Control. Autonomous Robots, 13, 21-35. https://doi.org/10.1023/A:1015674004201

Billingsley, J., Visala, A., \& Dunn, M. (2008). Robotics in Agriculture and Forestry. Handbook of Robotics (pp. 1065-1077). Berlin, Germany: Springer. https://doi.org/10.1007/978-3-540-30301-5_47

Bonadies, S., \& Gadsden, S. (2019). An Overview of Autonomous Crop Row Navigation Strategies for Unmanned Ground Vehicles. Journal of Engineering in Agriculture, Environment and Food, 12(1), 24-31. https://doi.org/10.1016/j.eaef.2018.09.001 
Bonadies, S., Smith, N., Niewoehner, N., Lee, A., Lefcourt, A., \& Gadsden, S. (2017). Development of Proportional-Integral-Derivative and Fuzzy Control Strategies for Navigation in Agricultural Environments. ASME Journal of Dynamic Systems, Measurement, and Control, 140(6). https://doi.org/10.1115/1.4038504

Bond, W., \& Grundy, A. (2001). Non-chemical weed management in organic farming systems. Weed Research, 41(5), 383-405. https://doi.org/10.1046/j.1365-3180.2001.00246.x

Bontsema, J., van Asselt, C., Lempens, P., \& van Straten, G. (1998). Intra-Row Weed Control: A Mechatronics Approach. IFAC Proceedings Volumes, 31(12), 93-97. https://doi.org/10.1016/S1474-6670(17)36047-0

Durant, D., Perumpral, J., \& Desai, C. (1980). Soil bin test facility for soil-tillage tool interaction studies. Soil and Tillage Research, 1, 289-298. https://doi.org/10.1016/0167-1987(80)90031-8

Green, J., \& Owen, M. (2011). Herbicide-Resistant Crops: Utilities and Limitations for Herbicide-Resistant Weed Management. Journal of Agriculture and Food Chemistry, 59(11), 5819-5829. https://doi.org/ $10.1021 / \mathrm{jf101286h}$

Ransom, J., \& Endres, G. (2014). Corn Growth and Management: Quick Guide (A1173). Fargo, North Dakota: North Dakota State University.

Sutton Agricultural Enterprises. (2020). Steketee Finger Weeder. Salinas, California: Sutton Agricultural Enterprises.

Taylor, J. E., Charlton, D., \& Yúnez-Naude, A. (2012). The End of Farm Labor Abundance. Applied Economics Perspectives and Policy, 34(4), 587-598. https://doi.org/10.1093/aepp/pps036

Upadhyaya, M., \& Blackshaw, R. (2007). Non-Chemical Weed Management: Principles, Concepts, and Technology. Oxfordshire, England: CABI. https://doi.org/10.1079/9781845932909.0000

Vidoni, R., Bietresato, M., Gasparetto, A., \& Mazzetto, F. (2015). Evaluation and Stability Comparison of Different Vehicle Configurations for Robotic Agricultural Operations on Side-Slopes. Biosystems Engineering, 129, 197-211. https://doi.org/10.1016/j.biosystemseng.2014.10.003

\section{Copyrights}

Copyright for this article is retained by the author(s), with first publication rights granted to the journal.

This is an open-access article distributed under the terms and conditions of the Creative Commons Attribution license (http://creativecommons.org/licenses/by/4.0/). 